\title{
Serum levels of anti-CCP antibodies, anti-MCV antibodies and RF IgA in the follow-up of patients with rheumatoid arthritis treated with rituximab
}

\author{
Martina Fabris $\bullet$ Salvatore De Vita $\bullet$ Nadia Blasone $\bullet$ Daniela Visentini • \\ Elena Pezzarini • Elena Pontarini • Cinzia Fabro • Luca Quartuccio • \\ Saulle Mazzolini • Francesco Curcio • Elio Tonutti
}

Received: 28 July 2010 / Accepted: 19 August 2010

(c) Springer-Verlag 2010

\begin{abstract}
Rheumatoid arthritis (RA) is characterized by the presence of circulating rheumatoid factor (RF) and anticitrullinated peptide antibodies (ACPA), which are positive in about $70-80 \%$ of patients. APCA have a higher specificity and therefore a higher diagnostic power than RF, but are less informative than RF in monitoring the course of the disease in patients under treatment. Recently, it has been reported that the anticitrullinated vimentin (a-MCV) antibody test can identify a particular subgroup of APCA that may be negative for anticyclic citrullinated peptide (a$\mathrm{CCP}$ ) antibodies. Concerning RF, the RF IgA isotype has been described as a more specific marker of erosive joint damage than total RF. The aim of our study was to monitor the levels of a-CCP, a-MCV, total RF and RF IgA in the follow-up of patients with RA treated with B-lymphocytedepletive rituximab (RTX), to detect any differences or peculiarities in patterns of these autoantibodies, especially in relation to their potential use as predictive markers of therapeutic response. We studied 30 patients with RA treated with RTX. All patients were previously unresponsive to
\end{abstract}

M. Fabris (更) $\cdot$ S. Mazzolini $\cdot$ F. Curcio

Clinical Pathology, Azienda Ospedaliero-Universitaria di Udine,

P.le S. Maria della Misericordia n.15, 33100 Udine, Italy

Tel.: +39-0432-552327

Fax: +39-0432-552402

E-mail: fabris.martina@aoud.sanita.fvg.it

N. Blasone $\cdot$ D. Visentini $\cdot$ E. Pezzarini $\cdot$ E. Tonutti

Immunopathology and Allergology,

Azienda Ospedaliero-Universitaria, Udine, Italy

S. De Vita - E. Pontarini - C. Fabro · L. Quartuccio

Rheumatology Clinic, Azienda Ospedaliero-Universitaria, Udine, Italy at least 6 months of therapy with disease-modifying antirheumatic drugs (DMARDs; methotrexate, leflunomide, cyclosporine, chloroquine) and/or at least 6 months of therapy with anti-TNF biologics. The evaluation of response to RTX was made at month +6 using the EULAR criteria (DAS28), a-CCP, a-MCV, total RF and RF IgA were determined at baseline (before the first infusion of RTX) and after 1, 3 and 6 months. In serum samples obtained before treatment two cytokines essential for Blymphocyte proliferation, interleukin 6 (IL-6) and B-lymphocyte stimulator (BLyS) were also determined. In all patients a significant and consistent reduction in all the tested antibodies was found during follow-up, with no differences in respect of the degree of response to RTX. Of note, at baseline, generally a higher titre of all autoantibodies was seen in patients who then showed a better response to RTX. Finally, there were no differences in serum concentrations of IL- 6 and BLyS in patients in relation to the presence or absence of the autoantibodies investigated, nor was there any significant correlation between the serum concentrations of the cytokines and the titres of the autoantibodies. Thus, neither a-MCV compared to a$\mathrm{CCP}$, nor RF IgA compared to routine total RF, provided any additional predictive information in the follow-up of patients with RA treated with RTX.

Keywords Anticitrullinated peptide antibodies Antimodified citrullinated vimentin antibodies Rheumatoid factor $\cdot$ Rheumatoid arthritis $\cdot$ Rituximab

\section{Introduction}

The B-lymphocyte-depletive drug rituximab (RTX), a chimeric anti-CD20 monoclonal antibody, was intro- 
duced in 2001 for the treatment of B-lymphocyte-mediated autoimmune diseases such as rheumatoid arthritis (RA), systemic lupus erythematosus (SLE), Sjögren's syndrome (SS) and cryoglobulinaemic syndrome [1-5]. The mechanism by which RTX depletes B-lymphocytes in the peripheral blood is not completely known. It is believed that the drug induces cytotoxicity through the activation of complement antibody cell-mediated reactions $[6,7]$. These reactions, however, explain only part of the mechanisms of action of RTX. For this reason it is believed that other molecular pathways may be activated by the drug (apoptosis, phagocytosis mediated by FcyR receptors) and may explain the variability in clinical response to RTX in several pathologies in which has been used [8, 9].

During the course of RA, RTX has been shown to produce an effective reduction of $\mathrm{CD} 20^{+} \mathrm{B}$-lymphocytes in both the blood and peripheral areas of inflammation and in the bone marrow which limits the function of antigen-presenting cells to $\mathrm{T}$ lymphocytes and the production of autoantibodies [9-11]. Clinical efficacy is accompanied by a reduction in the level of autoantibodies, without affecting that of total immunoglobulins [11], thus preserving the patient's immune capacity against infections and suggesting a selective effect on the B-cell population implicated in the pathogenesis of RA. A depletive activity against the CD20+ T-lymphocyte population has also recently been demonstrated, characterized by constitutive production of proinflammatory cytokines and a high state of activation [12].

Citrullinated peptide antibodies (APCA), in particular anticyclic citrullinated peptide (a-CCP) and rheumatoid factor (RF) are the most reliable serological markers for the diagnosis of patients with RA [13-15]. a-CCP appears to be highly specific for RA (95-97\%), with a sensitivity comparable to that of RF (75-80\%) and a more predictive role towards the erosive and rapidly progressive disease [16-18]. The role of a-CCP and RF in the monitoring of patients with RA treated with different therapeutic approaches is still a matter of debate. Recent studies have shown that the titre of a-CCP antibodies decreases partially after the first months of therapy and then remains stable in the long term regardless of the therapeutic response [19-21], while the reduction in RF appears to be more marked and more significantly correlated with the therapeutic response [21].

Among the proteins that are targeted by APCA, citrullinated vimentin soon became a very promising target because it is present specifically in the pannus and in the synovial fluid of RA patients $[22,23]$. Therefore, the industry has recently proposed a specific test for antihuman modified citrullinated vimentin (a-MCV) [24].
a-MCV were first described in 1989 and were called anti-Sa, the initials of the first patient in whom they were identified [25]. Numerous studies have been formalized using experimental and commercial tests for a-MCV. These studies have shown that the sensitivity of a-MCV antibodies is similar to that of a-CCP, and the specificity is slightly lower [26-29]. The predictive power in relation to radiographic progression and a high association with the shared epitope are even higher for a-MCV than for aCCP antibodies [30, 31], but for a-MCV major prognostic information is linked to a high titre and not to its mere presence [31]. The clinical role of a-MCV is still a matter of debate. It cannot replace a-CCP, but they can certainly occur together, because a small percentage of patients with RA are a-MCV-positive and a-CCP negative. Thus aMCV antibodies could be used as second level test to support the diagnosis of RA in patients with a strong suspicion who are negative for a-CCP and RF [26].

The RFs are a heterogeneous group of autoantibodies directed against antigenic determinants in the constant region (Fc fragment) of the heavy chain of $\mathrm{IgG}$. They may belong to all classes of antibodies, although IgM is the most representative [32]. RF IgA has recently been reassessed as a marker of RA, and it seems that RF IgA, as well as having a high predictive value in the diagnostic phase, shows a higher correlation with the development of bone erosion and extraarticular manifestations [33, 34].

Biologics have revolutionized the treatment of RA, being much more effective than traditional therapies. However, these drugs are expensive and are ineffective in $30-40 \%$ of patients [35-38]. The search for biological markers useful in identifying patients with a greater propensity to respond to different biological drugs, whose performance is related to therapeutic response, is a very important field of current research [39, 40].

Recently, the a-MCV antibodies have been investigated in the follow-up of patients with RA treated with antiTNF- $\alpha /$ drugs, and they appear to have added value compared to the classic assay of a-CCP and RF since a-MCV antibodies decrease more quickly and are associated with clinical improvement [26]. Nothing has yet been reported for treatment with RTX, a drug that is directed to the autoantibody secreting cells.

The aim of this study was to analyse the serum levels of a-MCV, a-CCP and total and IgA-specific RF in the follow-up of patients with RA treated with RTX to identify any differences or peculiarities in the patterns of these autoantibodies, especially in relation to their potential use as predictive markers of therapeutic response. In addition baseline serum levels of IL-6 and B-lymphocyte stimulator (BLyS) were also determined, as they are key cytokines in B-cell homeostasis [41, 42]. 


\section{Patients and methods}

Patients

The study was performed in a total of 54 patients with RA (10 men, 44 women; mean age $59.7 \pm 13$ years) diagnosed according to the criteria of the American College of Rheumatology (ACR) [43]. Their mean duration of disease was $13.8 \pm 10.3$ years. On entry, the patients were receiving treatment with disease-modifying antirheumatic drugs (DMARDs) and/or low doses of steroids ( $<10 \mathrm{mg} /$ day). Of these 54 patients, 30 were directed to treatment with RTX, in 13 due to lack of response to conventional DMARDs for at least 6 months and in 17 due to lack of response to antiTNF $\alpha$ drugs for at least 6 months. The RTX treatment consisted of two rounds of intravenous infusions of $1 \mathrm{~g}$ on day 0 and day 15. In patients receiving anti-TNF $\alpha$ drugs, a wash-out period of at least 1 month before treatment with RTX was observed. Clinical response was assessed after 6 months according to the criteria of the European League Against Rheumatisms (EULAR), using the Disease Activity score on 28 joints (DAS28).

In all patients treated with RTX a baseline serum sample, i.e. obtained during the days immediately preceding the first infusion of RTX, was analysed. In 22 patients, serum samples obtained 1 month, 3 months and 6 months after the first RTX infusion were also available and tested. All serum samples were stored at $-80^{\circ} \mathrm{C}$.

\section{Methods}

The serum levels of a-CCP, a-MCV and RF IgA were determined using automated enzyme-linked immunoassays (ELISA). In particular, the ELISA for the analysis of IgG a-CCP antibodies (Euro-Diagnostics) used highly purified synthetic peptides CCP-2. For the quantitative determination of a-MCV IgG antibodies an indirect solidphase ELISA was employed (Orgentec, Mainz, Germany).
For the determination of RF IgA the Orgentec ELISA was used. RF was determined by a routine turbidimetric method using an Olympus AU 2700 analyser. By this method, all the RF isotypes are detected, although RF IgM is the prevalent one, since $\operatorname{IgA}$ and $\mathrm{IgG}$ are often obscured by autoaggregation. We considered this test to determine total RF. Serum levels of IL-6 were determined using a capture immunofluorimetric method based on magnetic beads with chemiluminescent signal detection (Access 2 immunoassay system; Beckman Coulter, Fullerton, CA). The dosage of serum BLyS was determined using an ELISA (Quantikine Human BAFF/BLyS; R\&D Systems, Minneapolis, MN). All assays were performed at the Laboratory of Immunopathology and Allergology of the University Hospital of Udine.

\section{Statistical analysis}

The statistical analysis was done using the software GraphPad Prism (Intuitive Software for Science, San Diego, CA). The differences between groups of unpaired values were analysed using the nonparametric MannWhitney test, while paired values were compared using the Wilcoxon test. All values are expressed as means \pm standard deviation. Results were considered significant for $p<0.05$.

\section{Results}

Characteristics of a-MCV-positive and a-MCV-negative patients

In the group of 54 RA patients investigated, although the number was limited, we found one a-MCV-positive and a-CCP-negative patient, and one a-CCP-positive and aMCV-negative patient (Table 1). The prevalence among our study group was therefore comparable to that among

Table 1 Characteristics of a-MCV-positive and a-MCV-negative RA patients

\begin{tabular}{lrr}
\hline & a-MCV-positive $(n=36)$ & a-MCV negative $(n=18)$ \\
\hline a-CCP & & \\
$\quad$ Positive & $35 / 36(97.2 \%)$ & $1 / 18(5.6 \%)$ \\
$\quad$ Negative & $1 / 37(2.8 \%)$ & $17 / 18(94.4 \%)$ \\
Total RF & & \\
Positive & $32 / 36(88.9)$ & $3 / 18(16.7 \%)$ \\
Negative & $4 / 36(11.1 \%)$ & $15 / 18(83.3 \%)$ \\
RF IgA & & \\
Positive & $26 / 36(72.2 \%)$ & $2 / 18(11.1 \%)$ \\
Negative & $10 / 36(27.8 \%)$ & $16 / 18(88.9 \%)$ \\
BLyS ng/ml & $1.3 \pm 0.7$ & $1.4 \pm 0.6$ \\
IL-6 ng/ml & $38.3 \pm 46.0$ & $36.9 \pm 41.6$ \\
\hline
\end{tabular}


Table 2 Baseline prevalence of autoantibody seropositivity in the 30 RA patients treated with RTX in relation to the response according to the EULAR criteria at month +6

\begin{tabular}{lrrrr}
\hline Response & a-CCP & a-MCV & Total RF & RF IgA \\
\hline Good $(n=10)$ & $9(90 \%)$ & $9(90 \%)$ & $8(80 \%)$ & $6(60 \%)$ \\
Moderate $(n=14)$ & $12(85.7 \%)$ & $12(85.7 \%)$ & $13(92.8 \%)$ & $11(78.6 \%)$ \\
No response $(n=6)$ & $5(83.3 \%)$ & $5(83.3 \%)$ & $4(66.7 \%)$ & $3(50 \%)$ \\
\hline
\end{tabular}

previously published series [26]. The titre of the a-MCV antibodies showed a significant correlation with the titre of a-CCP $(R=0.83, p<0.0001)$ and that of total RF ( $R=0.46, p=0.0007)$ and RF IgA $(R=0.43, p=0.0013)$. No significant differences were seen in the levels of the cytokines BLyS and IL-6 in patients positive and negative for a-MCV (Table 1). Similarly, there were no significant differences in the levels of BLyS and IL-6 in patients positive and negative for RF IgA. Of note, in the 13 patients negative for all autoantibodies, serum levels of BLyS $(1.5 \pm 0.6 \mathrm{ng} / \mathrm{ml})$, a key factor in the proliferation of autoimmune B-cell clones, were similar to those of the seropositive patients with RA $(1.3 \pm 0.7 \mathrm{ng} / \mathrm{ml}, p$ not significant). To confirm these observations, there was no correlation between the titres of a-MCV, a-CCP, or total and IgA-specific RF or serum concentrations of BLyS and IL-6. Finally, there was no correlation between serum BLyS and IL-6 levels (data not shown).

Clinical response to RTX and baseline prevalence of autoantibodies

The assessment of clinical response to RTX after 6 months according to the EULAR criteria showed the following distribution: 6/30 (20\%) nonresponders, 14/30 (46.7\%) moderate responders, and 10/30 (33.3\%) good responders. As shown in Table 2, there were no significant differences in baseline prevalence of autoantibody positivity among the different subgroups of patients separated according to the EULAR response criteria found at 6 months.

Analysis of a-MCV, a-CCP, and total and IgA-specific RF serum levels during the follow-up of patients treated with RTX

In 22 of the 30 patients treated with RTX, it was possible to determine serum autoantibodies during the follow-up period. As shown in Fig. 1, overall, serum levels of a$\mathrm{CCP}$ and a-MCV were similarly significantly reduced during follow-up after treatment with RTX, although aCCP seemed to show a more marked and early reduction after 1 month compared to a-MCV. Finally, the levels of total and IgA-specific RF during follow-up showed a significant and entirely comparable reduction (Fig. 2).

As shown in Table 3, dividing patients according to level of response to therapy ( 7 with a good response, 13 with a moderate response, and 2 with no response)

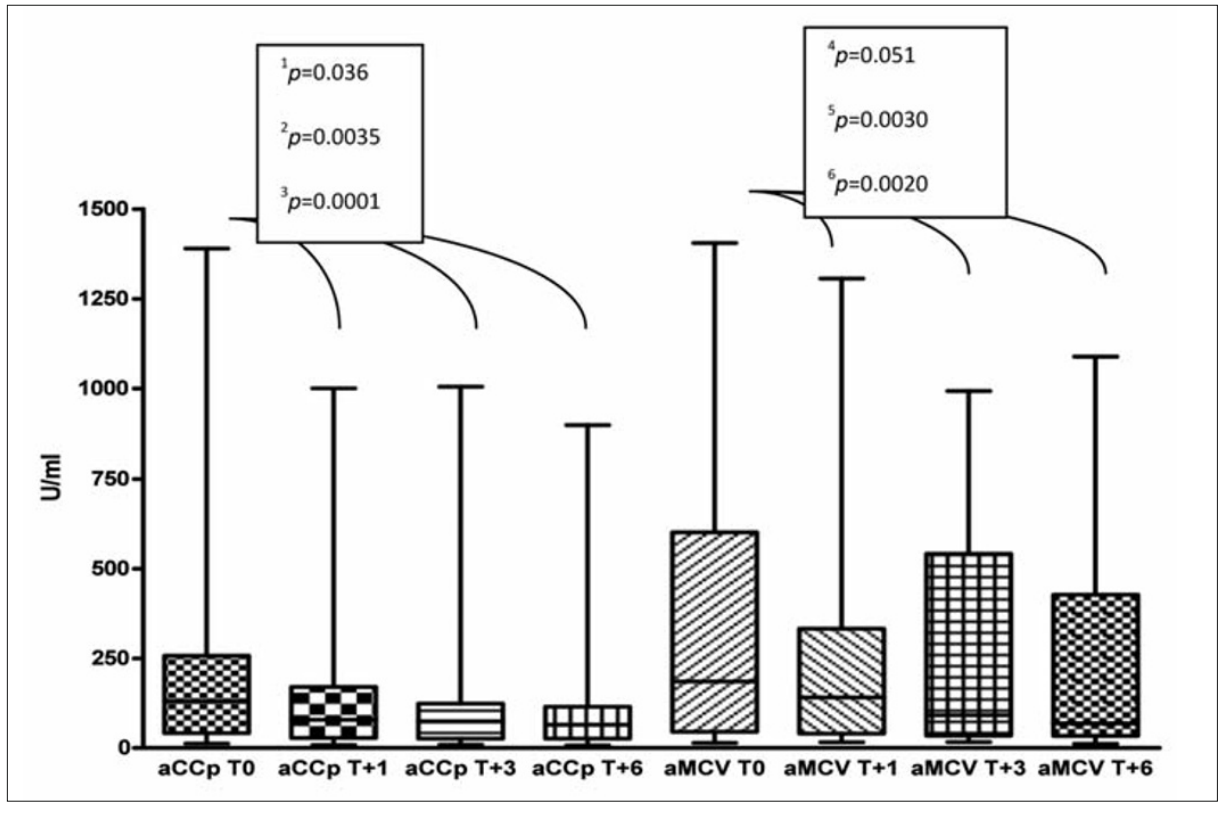

Fig. 1 Serum levels of a-CCP and aMCV antibodies during the follow-up of patients treated with RTX. The mean levels of a-CCP and a-MCV antibodies are shown with the boxes representing the interquartile ranges and the lines representing the 10th and 90th percentiles of the distribution of values at the given times (from $T 0$, baseline, to $T+6,6^{\text {th }}$ month). Baseline levels of aCCP were significantly reduced at month $+1\left({ }^{l} p\right)$ and even more at months $+3\left({ }^{2} p\right)$ and $+6\left({ }^{3} p\right)$. The reduction in aMCV serum levels was of borderline significance at month $+1 \quad\left({ }^{4} p\right)$, but showed increased significance at month $+3\left({ }^{5} p\right)$ and month $+6\left({ }^{6} p\right)$ 
Fig. 2 Serum levels of total and IgAspecific RF during the follow-up of patients treated with RTX. The mean levels of total and IgA-specific RF are shown with the boxes representing the interquartile ranges and the lines representing the 10th and 90th percentiles of the distribution of values at the given times (from $T 0$, baseline, to $T+66^{\text {th }}$ month). The levels of total RF were significantly reduced as early as at month $+1\left({ }^{l} p\right)$, then the reduction is maintained at month $+3\left({ }^{2} p\right)$ and month $+6\left({ }^{3} p\right)$. The reduction in RF IgA serum levels is less significant at month $+1\left({ }^{4} p\right)$ than at month $+3\left({ }^{5} p\right)$ and month $+6\left({ }^{6} p\right)$ $R F$, rheumatoid factor

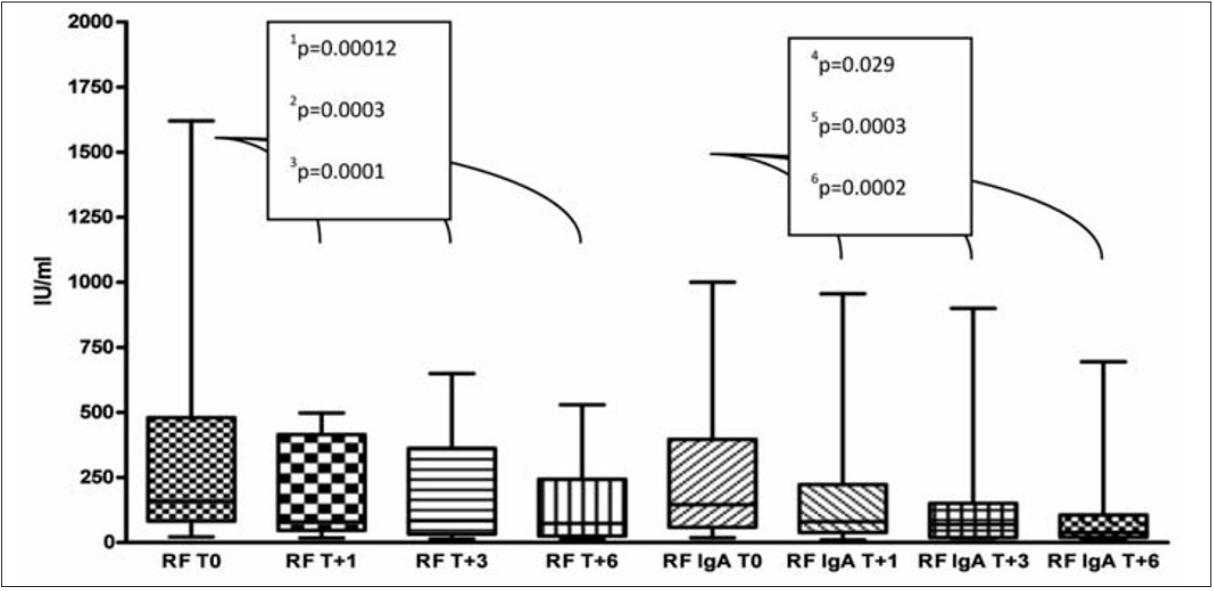

Table 3 Changes in serum levels of autoantibodies during the follow-up after RTX treatment in relation to the response according to the EULAR criteria at month +6

\begin{tabular}{lllll}
\hline & EULAR response & Baseline & Month +3 & Month +6 \\
\hline a-MCV $(\mathrm{IU} / \mathrm{ml})$ & Good $(n=7)$ & $552 \pm 545$ & $436 \pm 470$ & $422 \pm 434$ \\
& Moderate $(n=13)$ & $275 \pm 375$ & $154 \pm 264$ & $142 \pm 272$ \\
& No response $(n=2)$ & $138 \pm 67$ & $141 \pm 142$ & $166 \pm 181$ \\
a-CCP $(\mathrm{IU} / \mathrm{ml})$ & Good $(n=7)$ & $363 \pm 487$ & $249 \pm 308$ & $188 \pm 221$ \\
& Moderate $(n=13)$ & $195 \pm 265$ & $137 \pm 265$ & $114 \pm 231$ \\
& No response $(n=2)$ & $97 \pm 52$ & $84 \pm 87$ & $88 \pm 57$ \\
Total RF $(\mathrm{IU} / \mathrm{ml})$ & Good $(n=7)$ & $280 \pm 208$ & $226 \pm 246$ & $196 \pm 176$ \\
& Moderate $(n=13)$ & $436 \pm 547$ & $205 \pm 218$ & $166 \pm 182$ \\
RF IgA $(\mathrm{IU} / \mathrm{ml})$ & No response $(n=2)$ & $60 \pm 43$ & $25 \pm 16$ & $37 \pm 36$ \\
& Good $(n=7)$ & $317 \pm 459$ & $257 \pm 429$ & $209 \pm 325$ \\
& Moderate $(n=13)$ & $257 \pm 264$ & $113 \pm 107$ & $75 \pm 81$ \\
& No response $(n=2)$ & $152 \pm 115$ & $59 \pm 60$ & $46 \pm 35$ \\
\hline
\end{tabular}

Table 4 Changes in serum levels of autoantibodies during the follow-up after RTX treaemtn in a patient with longstanding remission after one single course of infusions

\begin{tabular}{|c|c|c|c|c|c|c|c|}
\hline & \multirow[t]{2}{*}{ Baseline } & \multicolumn{6}{|c|}{ Month } \\
\hline & & 1 & 3 & 6 & 12 & 24 & 48 \\
\hline $\mathrm{a}-\mathrm{CCP}(\mu \mathrm{I} / \mathrm{ml})$ & 44.7 & 77.4 & 75.5 & 66.1 & 35.1 & 78.2 & 143.6 \\
\hline a-MCV (IU/ml) & 906.8 & 888.2 & 861.3 & 899.7 & 652.7 & 1048.9 & 1513.1 \\
\hline Total RF (IU/ml) & 157.4 & 114.9 & 101.5 & 115.5 & 79.0 & 107.0 & 162.0 \\
\hline RF IgA (IU/ml) & 17.9 & 21.0 & 19.2 & 22.9 & 12.5 & 18.6 & 18.1 \\
\hline
\end{tabular}

revealed no significant differences. In all patients all the autoantibodies showed a marked reduction. However, patients with a good response tended to show more elevated serum levels of all the autoantibodies at time 0 , and therefore also at the subsequent time points.

Finally, the trend in the titre of autoantibodies is particularly exemplified in a patient who showed a good response after one cycle of RTX infusions. At the time of this report this patient had remained in clinical and laboratory remission for 4 years. At month 48 the levels of a-CCP and aMCV were even higher than at baseline, and also total RF, that fell during the first year (but never became negative), had returned to the baseline level by month 48 (Table 4). This patient was always RF IgA-negative.

\section{Discussion}

The diagnostic role of a-CCP antibodies and RF in RA has been well established for years [13]. However, a significant proportion of patients are seronegative for RA. Thus the search for new markers is a relevant and open problem, both from the pathogenetic and the clinical/diagnostic point of view. In this regard, the recent appreciation of the subclass of the ACPA, a-MCV, has aroused much interest, especially in relation to biological therapies, such as possibly more specific and sensitive markers of a-CCP. Tests for a-CCP using the second generation citrullinated antigen (CCP2) have shown an extremely high diagnostic performance and predictive 
value [44, 45]. But in some early studies a-MCV appeared more sensitive than a-CCP antibodies, although less specific [26, 46, 47]. However, more recent metaanalyses [28, 29] have shown a total overlapping diagnostic power between a-CCP and a-MCV testing, leading to the proposal of a-MCV analysis as a second-level test in selected cases of patients with a strongly suspected diagnosis of RA and who are negative for both a-CCP and RF. As we confirmed in our limited series, there is a small group of a-CCP-negative patients (about 10\%) with a significant titre of a-MCV, and an equal proportion of a-MCV-negative patients who are positive for a-CCP.

Although the diagnostic power appeared equivalent, there were some important differences in the prognostic power. The positive correlation between ACPA and the erosive evolution of joint damage, associated with the presence of the shared epitope, was higher for a-MCV than for a-CCP, especially if a-MCV was present at high titre [31]. In monitoring of therapeutic response, a-CCP has never proved very useful because it also shows a reduction in patients with an optimal response, and is always partial and uninformative [48]. However, a recent study has shown an earlier decline in a-MCV antibodies (month +18 ) than in aCCP antibodies (month +24 ) during treatment with infliximab, and also the titre of a-MCV antibodies seemed to follow significantly the clinical response scores [26], which was not seen with a-CCP antibodies.

Our study is the first to show the behaviour of aMCV and a-CCP antibody serum levels in the follow-up of RA patients treated with RTX. Contrary to what has been reported for infliximab, in our series of patients treated with RTX, the reduction in titre of a-MCV antibodies followed and did not precede that of a-CCP antibodies, and no differences were seen in relation to clinical response.

The choice of treatment and monitoring of treatment of RA with biological agents is currently a field of major interest, but strong data on biological markers still remain undefined [49-51]. Several authors have shown that the presence of RF, which is associated with more severe disease and a reduced therapeutic response in general, is a marker of a higher degree of response to RTX [50-52]. However, changes in RF serum levels in the follow-up after RTX treatment is not predictive of response, as we also found in this study, even focusing on $\operatorname{IgA}$-specific RF.

In our series as in others, if RF showed, albeit very rarely, a negative titre after treatment, a-CCP antibodies usually showed an initial reduction followed by stabilization [53]. A particular example in this respect was the patient who remained in clinical remission over 4 years of follow-up after a single cycle of RTX. In this patient a-CCP antibodies, a-MCV antibodies and RF showed a reduction which lasted until the month +12 , but then showed an increase to values higher than at baseline by month +48 . It is possible that the antigenic trigger and/or autoantigens that drive the autoantibody response remain or reappear after the therapeutic depletion of $\mathrm{CD} 20^{+} \mathrm{B}$ cells obtained with RTX. Rehnberg et al. [10] have recently stressed that the reduction in $\mathrm{CD} 27^{+} \mathrm{B}$ cells observed both in peripheral blood and in bone marrow of patients treated with RTX will not prevent the reappearance in the long term of autoantibodies, suggesting that mechanisms regulating autoantibody production are not destroyed by RTX, despite its clinical efficacy.

Evidence in the literature indicates that people with RA positive for RF IgA show more aggressive disease [33, 34]. Furthermore, RF IgA seems to have a greater role in predicting the development of RA than RF IgM. In our series, the positivity of RF IgA was not associated with response to RTX, nor did the changes in RF IgA serum levels differ significantly from those of total RF during the follow-up after treatment with RTX.

The analysis of two key cytokines involved in B-cell proliferation (IL-6 and BLyS) did not show significant differences between patients positive and negative for RF IgA. Therefore the increased severity of disease associated with the presence of RF IgA seems not to be attributable to these cytokines [33, 34]. Accordingly, no correlation was found between serum IL-6 or BLyS levels and the levels of total or IgA-specific RF. Similarly, no correlation was found between a-CCP and a-MCV serum levels and cytokine levels. Finally, the small group of patients seronegative for all autoantibodies tested did not show BLyS and IL-6 serum levels different from the rest of the patients studied. These results suggest that there is no direct relationship between levels of BLyS and IL-6 as markers of B-cell proliferation and B-cell autoantibody secretion in RA patients. Except for Sjögren's syndrome [54], a significant correlation between BLyS and autoantibodies is rarely in found in RA as in other systemic autoimmune diseases [55], as we have observed in mixed cryoglobulinaemic syndrome [56] and in an extended RA series (unpublished personal observations). Local, rather than systemic levels of both autoantibodies and pivotal disease cytokines could be more representative of disease activity in RA [57].

Of particular relevance was that patients with a better clinical response to RTX always tended to show, both at baseline and during follow-up, the highest levels of all autoantibodies tested. This observation is consistent with recently reported findings regarding the association between a positive response to RTX and RF and/or a-CCP positivity [50-52]. It can be assumed that RA patients characterized by greater autoantibody production respond better to therapies directed against $\mathrm{B}$ lymphocytes. 
In conclusion, we can say that the analysis of a-MCV antibodies or RF IgA did not appear to provide further information than that obtained by the currently performed analysis of a-CCP antibodies and routine RF in monitoring the response to RTX therapy in RA.

Conflict of interest statement The authors declare that they have no conflict of interest related to the publication of this article.

\section{References}

1. De Vita S, Zaja F, Sacco S et al (2002) Efficacy of selective B cell blockade in the treatment of rheumatoid arthritis: evidence for a pathogenetic role of B cells. Arthritis Rheum 46:2029-2033

2. Edwards JC, Leandro MJ, Cambridge G (2002) B-lymphocyte depletion therapy in rheumatoid arthritis and other autoimmune disorders. Biochem Soc Trans 30:824-828

3. Leandro MJ, Edwards JC, Cambridge G et al (2002) An open study of B lymphocyte depletion in systemic lupus erythematosus. Arthritis Rheum 46:2673-2677

4. Meijer JM, Pijpe J, Vissink A et al (2009) Treatment of primary Sjogren syndrome with rituximab: extended follow-up, safety and efficacy of retreatment. Ann Rheum Dis 68:284-285

5. De Vita S, Quartuccio L, Fabris M (2007) Rituximab in mixed cryoglobulinemia: increased experience and perspectives. Dig Liver Dis 39(Suppl 1):S122-S128

6. Taylor RP, Lindorfer MA (2008) Immunotherapeutic mechanisms of anti-CD20 monoclonal antibodies. Curr Opin Immunol 20:444-449

7. Maloney DG, B. Smith AR (2002) Rituximab: mechanism of action and resistance. Semin Oncol 29(Suppl 2):2-9

8. Uchida J, Hamaguchi Y, Oliver JA et al (2004) The innate mononuclear phagocyte network depletes B lymphocytes through Fc receptor-dependent mechanisms during anti-CD20 antibody immunotherapy. J Exp Med 199:1659-1669

9. Szodoray P, Alex P, Dandapani V et al (2004) Apoptotic effect of rituximab on peripheral blood B cells in rheumatoid arthritis. Scand J Immunol 60:209-218

10. Rehnberg M, Amu S, Tarkowski A et al (2009) Short- and long-term effects of anti-CD20 treatment on B cell ontogeny in bone marrow of patients with rheumatoid arthritis. Arthritis Res Ther 11:R123

11. Ziswiler HR, Aeberli D, Villiger PM, Möller B (2009) High-resolution ultrasound confirms reduced synovial hyperplasia following rituximab treatment in rheumatoid arthritis. Rheumatology (Oxford) 48:939-943

12. Wilk E, Witte T, Marquardt N et al (2009) Depletion of functionally active $\mathrm{CD} 20+\mathrm{T}$ cells by rituximab treatment. Arthritis Rheum 60:3563-3571

13. Nishimura K, Sugiyama D, Kogata Y et al (2007) Meta-analysis: diagnostic accuracy of anti-cyclic citrullinated peptide antibody and rheumatoid factor for rheumatoid arthritis. Ann Intern Med 146:797-808

14. Visser H, le Cessie S, Vos K et al (2002) How to diagnose rheumatoid arthritis early. A prediction model for persistent (erosive) arthritis. Arthritis Rheum 46:357-365

15. Rantapää-Dahlqvist S, de Jong BA, Berling E et al (2003) Antibodies against cyclic citrullinated peptide and IgA rheumatoid factor predict the development of rheumatoid arthritis. Arthritis Rheum 48:2741-2749

16. Kastbom A, Strandberg G, Lindroos A et al (2004) Anti-CCP antibody test predicts the disease course during three years in early rheumatoid arthritis (the TIRA project). Ann Rheum Dis 63:1085-1089
17. Chan MT, Owen P, Dunphy J et al (2005) Anti-cyclic citrullinated peptide antibodies are associated with erosive arthritis in SLE. Arthritis Rheum 52:S611

18. Szodoray P, Szabó Z, Kapitány A et al (2010) Anti-citrullinated protein/peptide autoantibodies in association with genetic and environmental factors as indicators of disease outcome in rheumatoid arthritis. Autoimmun Rev 9:140-143

19. Vallbracht I, Rieber J, Oppermann M et al (2004) Diagnostic and clinical value of anti-citrullinated peptide antibodies compared with rheumatoid factor isotypes in rheumatoid arthritis. Ann Rheum Dis 63:1079-1084

20. Bobbio-Pallavicini F, Alpini C, Caporali R et al (2004) Autoantibody profile in rheumatoid arthritis during long-term infliximab treatment. Arthritis Res Ther 6:R264-R272

21. Alessandri C, Bombardieri M, Papa N et al (2004) Decrease of anti-cyclic citrullinated peptide antibodies and rheumatoid factor following ant-TNFalpha therapy (infliximab) in rheumatoid arthritis is associated with clinical improvement. Ann Rheum Dis 63:1218-1221

22. Bang H, Egerer K, Gauliard A et al (2007) Mutation and citrullination modifies vimentin to a novel autoantigen for rheumatoid arthritis. Arthritis Rheum 56:2503-2511

23. Snir O, Widhe M, Hermansson M et al (2010) Antibodies to several citrullinated antigens are enriched in the joints of rheumatoid arthritis patients. Arthritis Rheum 62:44-52

24. Mutlu N, Bicakcigil M, Tasan DA et al (2009) Comparative performance analysis of 4 different anti-citrullinated protein assays in the diagnosis of rheumatoid arthritis. J Rheumatol 36:491-500

25. Vossenaar R, Despres N, Lapointe E et al (2004) Rheumatoid arthritis specific anti-Sa antibodies target citrullinated vimentin, Arthritis Res Ther 6:R142-R150

26. Nicaise Roland P, Grootenboer Mignot S, Bruns A et al (2008) Antibodies to mutated citrullinated vimentin for diagnosing rheumatoid arthritis in anti-CCP-negative patients and for monitoring infliximab therapy. Arthritis Res Ther 10:R142

27. van der Linden MP, van der Woude D, Ioan-Facsinay A et al (2009) Value of anti-modified citrullinated vimentin and thirdgeneration anti-cyclic citrullinated peptide compared with second-generation anti-cyclic citrullinated peptide and rheumatoid factor in predicting disease outcome in undifferentiated arthritis and rheumatoid arthritis. Arthritis Rheum 60:2232-2241

28. Qin X, Deng Y, Xu J et al (2010) Meta-analysis: diagnostic value of serum anti-mutated citrullinated vimentin antibodies in patients with rheumatoid arthritis. Rheumatol Int. DOI: 10.1007/s00296-009-1343-3

29. Luime JJ, Colin EM, Hazes JM, Lubberts E (2010) Does antimutated citrullinated vimentin have additional value as a serological marker in the diagnostic and prognostic investigation of patients with rheumatoid arthritis? A systematic review. Ann Rheum Dis 69:337-344

30. Mathsson L, Mullazehi M, Wick MC et al (2008) Antibodies against citrullinated vimentin in rheumatoid arthritis: higher sensitivity and extended prognostic value concerning future radiographic progression as compared with antibodies against cyclic citrullinated peptides. Arthritis Rheum 58:36-42

31. Syversen SW, Goll GL, van der Heijde D et al (2010) Prediction of radiographic progression in rheumatoid arthritis and the role of antibodies against mutated citrullinated vimentin: results from a 10-year prospective study. Ann Rheum Dis 69:345-351

32. Robecchi A, Daneo V (1959) Research on the behaviour, nature and meaning of the rheumatoid factor. Acta Rheumatol Scand 5:245-262

33. Berling E, Johansonn T, Sundin U et al (2006) Radiological outcome in rheumatoid arthritis is predicted by presence of antibodies against cyclic citrullinated peptide before and at disease onset, and by $\operatorname{IgA}-\mathrm{RF}$ at disease onset. Ann Rheum Dis 65:453-458 
34. Bobbio-Pallavicini F, Caporali R, Alpini C et al (2007) High IgA rheumatoid factor levels are associated with poor clinical response to tumour necrosis factor alpha inhibitors in rheumatoid arthritis. Ann Rheum Dis 66:302-307

35. Sfikakis PP (2010) The first decade of biologic TNF antagonists in clinical practice: lessons learned, unresolved issues and future directions. Curr Dir Autoimmun 11:180-210

36. Perosa F, Prete M, Racanelli V, Dammacco F (2010) CD20depleting therapy in autoimmune diseases: from basic research to the clinic. J Intern Med 267:260-277

37. Buch MH (2010) Sequential use of biologic therapy in rheumatoid arthritis. Curr Opin Rheumatol 22:321-329

38. Benucci M, Li Gobbi F, Sabadini L et al (2009) The economic burden of biological therapy in rheumatoid arthritis in clinical practice: cost-effectiveness analysis of sub-cutaneous antiTNFalpha treatment in Italian patients. Int $\mathrm{J}$ Immunopathol Pharmacol 22:1147-1152

39. Dörner T, Kinnman N, Tak PP (2010) Targeting B cells in immune-mediated inflammatory disease: a comprehensive review of mechanisms of action and identification of biomarkers. Pharmacol Ther 125:464-475

40. Tas SW (2009) Personalised treatment of arthritis in the next eRA. Neth J Med 67:362-363

41. Kishimoto T (2006) Interleukin-6: discovery of a pleiotropic cytokine. Arthritis Res Ther 8(Suppl 2):S2

42. Mackay F, Mackay CR (2002) The role of BAFF in B-cell maturation, T-cell activation and autoimmunity. Trends Immunol 23:113-115

43. Arnett FC, Edworthy SM, Bloch DA et al (1988) The American Rheumatism Association 1987 revised criteria for the classification of rheumatoid arthritis. Arthritis Rheum 31:315-324

44. van Gaalen FA, Visser H, Huizinga TW (2005) A comparison of the diagnostic accuracy and prognostic value of the first and second anti-cyclic citrullinated peptides autoantibody (CCP1 and CCP2) tests for rheumatoid arthritis. Ann Rheum Dis 64:1510-1512

45. Bizzaro N, Tonutti E, Tozzoli R et al (2007) Analytical and diagnostic characteristics of 11 second- and third-generation immunoenzymatic methods for the detection of antibodies to citrullinated proteins. Clin Chem 53:1527-1533

46. Mathsson L, Mullazhei M, Wick MC et al (2008) Antibodies against citrullinated vimentin in rheumatoid arthritis: higher sensitivity and extended prognostic value concerning future radiographic progression as compared with antibodies against cyclic citrullinated peptides. Arthritis Rheum 58:36-45
47. Damjanovska L, Thabet MM, Leverht EW et al (2010) The diagnostic value of anti-MCV antibodies in differentiating early inflammatory arthritis. Ann Rheum Dis 69:730-732

48. De Rycke L, Verhelst X, Kruithof E et al (2005) Rheumatoid factor, but not anti-cyclic citrullinated peptide antibodies, is modulated by infliximab treatment in rheumatoid arthritis. Ann Rheum Dis 64:299-302

49. Thurlings RM, Vos K, Wijbrandts CA et al (2008) Synovial tissue response to rituximab: mechanism of action and identification of biomarkers of response. Ann Rheum Dis 67:917-925

50. Quartuccio L, Fabris M, Salvin S et al (2009) Rheumatoid factor positivity rather than anti-CCP positivity, a lower disability and a lower number of anti-TNF agents failed are associated with response to rituximab in rheumatoid arthritis. Rheumatology (Oxford) 48:1557-1559

51. Pyrpasopoulou A, Douma S, Triantafyllou A et al (2010) Response to rituximab and timeframe to relapse in rheumatoid arthritis patients: association with B-cell markers. Mol Diagn Ther 14:43-48

52. van Vollenhoven RF, Chatzidionysiou K, Gabay C et al (2009) Rheumatoid factor predicts response to rituximab in a European registry-based cohort: 6-month results from the collaborative European registries for rituximab in rheumatoid arthritis (CERERRA). Ann Rheum Dis 68(Suppl3):579

53. Bruns A, Nicaise-Roland P, Hayem G et al (2009) Prospective cohort study of effects of infliximab on rheumatoid factor, anticyclic citrullinated peptide antibodies and antinuclear antibodies in patients with long-standing rheumatoid arthritis. Joint Bone Spine 76:248-253

54. Mariette X, Roux S, Zhang J et al (2003) The level of BLyS (BAFF) correlates with the titre of autoantibodies in human Sjögren's syndrome. Ann Rheum Dis 62:168-171

55. Becker-Merok A, Nikolaisen C, Nossent HC (2006) B-lymphocyte activating factor in systemic lupus erythematosus and rheumatoid arthritis in relation to autoantibody levels, disease measures and time. Lupus 15:570-576

56. Fabris M, Quartuccio L, Sacco S et al (2007) B-Lymphocyte stimulator (BLyS) up-regulation in mixed cryoglobulinaemia syndrome and hepatitis-C virus infection. Rheumatology (Oxford) 46:37-43

57. Tan SM, Xu D, Roschke V et al (2003) Local production of B lymphocyte stimulator protein and APRIL in arthritic joints of patients with inflammatory arthritis. Arthritis Rheum 48:982-992 